\title{
Identifying deafness in early childhood: requirements after the newborn hearing screen
}

\author{
PM Watkin, M Baldwin
}

\section{Correspondence to \\ P M Watkin, Consultant in Audiological Medicine, Whipps Cross University Hospital, Leytonstone, London E11 1NR, UK; peter.watkin@whippsx.nhs.uk}

Accepted 28 July 2010 Published Online First 2 November 2010

\begin{abstract}
Background Newborn hearing screening has been nationally implemented, but longitudinal cohort follow-up is required to inform Children's Hearing Services of the requirements for postneonatal care pathways.

Methods A 10-year cohort of 35668 births enrolled into a Universal Neonatal Hearing Screen was followed up until the children had completed the first year of primary school.
\end{abstract}

Results There were 3.65/1000 children with a permanent hearing impairment of any degree embarking on their education. 1.51/1000 had a moderate or worse bilateral deafness but only 0.9/1000 with this degree of deafness had been identified by newborn screening. Postneonatal care pathways were required to identify those with congenital impairments missed by the screen $(0.11 / 1000)$, those moving into the district $(0.25 / 1000)$ and those with late onset deafness $(0.25 / 1000)$. An additional postneonatal yield of 1.2/1000 had mild or unilateral impairments. When all degrees of impairment were considered $51 \%$ of the children with a permanent hearing impairment had required identification by postneonatal care pathways.

Conclusions Despite the success of the newborn hearing screen, the provision of postneonatal pathways remains essential for identifying deafness in early childhood.

\section{INTRODUCTION}

The NHS newborn hearing screening programme (NHSP) has been nationally implemented since 2006 and has been justifiably considered a 'quite astonishing achievement'. ${ }^{1}$ However, expectations should remain realistic and concerns have been expressed about the maintenance of services required to identify children with deafness not picked up by the screen. ${ }^{2}$ Two universal neonatal hearing screens (UNHS) initially implemented in the $\mathrm{UK}^{3}{ }^{4}$ and the NHSP subsequently introduced have all achieved similar yields, but the possible prevalence of deafness in school children has been estimated to be twice their yields, which suggests that many cases of deafness require picking up later in childhood. ${ }^{5}$ Longitudinal cohort follow-up in early childhood is needed to confirm this.

Case finding in early childhood after the newborn screen is through care pathways reactive to professional or parental concerns with a backstop school entry screen (SES) still retained in the majority of districts. ${ }^{6}$ The pathways have been addressed by the Department of Health Guide for Transforming Services for Children with Hearing Difficulty. ${ }^{7}$ This recommends postneonatal assessments and surveillance by local Children's Hearing Services, led by a paediatrician, with a

\section{What is already known on this topic}

- The newborn hearing screening programme has been rolled out across the country and the highest performing sites have identified $0.96 / 1000$ with bilateral and $0.49 / 1000$ with unilateral permanent congenital hearing impairment that is moderate or worse in degree.

- National ascertainment of deaf children has demonstrated that the prevalence of bilateral deafness by 9 years of age has risen to $1.65 / 1000$ and could be as high as $2 / 1000$, but the most effective way of identifying these additional children is currently unclear.

\section{What this study adds}

- Less than $60 \%$ of children with moderate or worse bilateral deafness in the initial years of their primary education have been identified neonatally even when an established and sensitive newborn hearing screen is in place.

- Reactive care pathways responsive to parental and professional concerns are currently the most effective way of picking up children with hearing impairment not identified through newborn screening and they should remain an essential and maintained children's hearing service.

network of specialist services seeing more complex cases and providing the assessments and managements from the newborn screen. This vision was developed from the Royal College of Paediatrics and Child Health Working Group Review of Children's Audiology Services. ${ }^{8}$ However, it had not been informed by longitudinal evaluations of cohorts that had received the newborn screen. Good quality cumulative data will eventually be available from NHSP to guide this. However, following a cohort over an extended period is a challenge more readily met by a local screen. Follow-up of a cohort enrolled into the Whipps Cross University Hospital UNHS provides this opportunity.

The Whipps Cross UNHS employed a twostage Transient Otoacoustic Emission (TEOAE) test to screen the cochlear function of the newborn cohort. It provided a service in the East 
London Borough of Waltham Forest from 1992 and was subject to on-going evaluations and secular developments. ${ }^{3} 9$ Along with the Wessex controlled trial it informed the critical review of neonatal hearing screening which led to the NHSP implementation. ${ }^{10}$ In 2002, the Whipps Cross screen became a pilot site of the national programme and underwent some modifications. Specificity was improved by undertaking automated auditory brainstem response (A-ABR) when TEOAEs could not be recorded. ${ }^{11}$ Additionally screening both neural and cochlear responses was introduced in the special care baby unit to identify auditory neuropathy spectrum disorder (ANSD) where cochlear function is retained but auditory disorder results from neural dysfunction. ${ }^{12}$ Earlier screen completion was also encouraged. Nevertheless, the Whipps Cross screen had accurately predicted the sensitivity of the newborn screen. The yield for the highest performing NHSP sites has been $0.96 / 1000$ for bilateral and 0.49/1000 for unilateral permanent congenital hearing impairment (PCHI) and this is essentially the same as the Whipps Cross yields reported a decade earlier. It is this sensitivity which governs the need for later case identification.

The Waltham Forest cohort has been longitudinally followed up to investigate the need for postneonatal case finding. Comparing the local case ascertainment with national predictions of prevalence has been undertaken so that findings from this local evidence base can be generalised. The Audiology Service has remained stable, and joint working with children's services, education and ENT has optimised ascertainment. Postneonatal case finding is presented from a 10-year Waltham Forest cohort which received a newborn hearing screen and was followed up until it had completed the first year of primary school.

\section{METHODS}

\section{The cohort enrolled for follow-up}

The cohort followed up was born from September 1992, when UNHS coverage had been optimised, until 2002 when NHSP was introduced. The UNHS was available for all Waltham Forest residents over the 10 years. They also had the opportunity to receive the SES and surveillance during their first year of school. There had also been a lag of several months to allow full assessment of the youngest in the cohort.

\section{Postneonatal care pathways}

The Waltham Forest cohort was enrolled in the community child health programmes. The health visitor distraction test (HVDT) was offered as a universal screen at 7 months for the initial 5 years with a targeted HVDT subsequently introduced until the NHSP protocol was adopted. Hearing surveillance was undertaken in primary care and the SES was retained. The audiology service received referrals from all sources and directly from parents. All PCHIs in the district were notified through the education service.

\section{Data analysis}

Results from the UNHS and postneonatal pathways were recorded on a database constructed for this purpose. Degrees of impairment were described by four frequency averages from 0.5 to $4 \mathrm{kHz}$ with the degree in the better hearing ear (BHE) used in bilateral impairments, and in the worse hearing ear (WHE) when one ear had an impairment and the other average thresholds of $<20 \mathrm{~dB}$ hearing level (HL). The yield of cases with a moderate or worse PCHI is routinely reported by NHSP and the current analyses employed this descriptor by aggregating those with an average threshold $\geq 40 \mathrm{~dB}$ HL. Ascertainment by school age was compared with the Medical Research Council (MRC) Institute of Hearing Research studies of hearing impaired children in the Trent region and of the prevalence of PCHI in the UK. ${ }^{5}{ }^{13}$ Distributions of hearing impairments in the cohorts were compared using Pearson's $\chi^{2}$. Definitions were based on those used by the MRC and are detailed in the glossary. Median and interquartile ages were used when the children were referred or had their impairment confirmed with the 75 th centile used as the index of late referrals.

\section{Ethics committee}

The study was considered a longitudinal service evaluation (NHS National Research Ethics Services).

\section{RESULTS}

\section{The prevalence of $\mathrm{PCHI}$ in primary school}

From 1992 to 2002, a cohort of 35668 had been enrolled into the community child health programmes with 33860 $(95 \%)$ having received a newborn hearing screen. By the time the cohort had passed through the first year of school, 130 children had been identified with either a bilateral or unilateral PCHI (3.64/1000). The prevalence by degree of hearing impairment is detailed in table 1 . Of the 54 children with moderate and worse bilateral PCHI, 30 had moderate deafness (0.84/1000), 12 severe PCHI (0.34/1000) and another 12 profound deafness $(0.34 / 1000)$. Of the 29 with unilateral hearing loss (UHL), 24 (0.67/1000) had moderate and worse deafness in their WHE.

Table 1 The factors affecting the prevalence of PCHI in primary school $(n=130)$

\begin{tabular}{|c|c|c|c|c|}
\hline & Congenital PCHI born in district & PCHls moved in & Late onset PCHI & PCHI in primary school \\
\hline \multicolumn{5}{|c|}{ Moderate and worse bilateral ( $\geq 40 \mathrm{~dB} \mathrm{HL}$ in BHE) } \\
\hline Number & 36 & 9 & 9 & 54 \\
\hline Prevalence/1000 (95\% Cl) & 1.01 (0.68 to 1.34$)$ & 0.25 (0.09 to 0.42$)$ & 0.25 (0.09 to 0.42 ) & 1.51 (1.11 to 1.92$)$ \\
\hline \multicolumn{5}{|c|}{ Mild bilateral (20-39 dB HL in BHE) } \\
\hline Number & 35 & 4 & 8 & 47 \\
\hline Prevalence/1000 (95\% Cl) & 0.98 (0.66 to 1.31 ) & $0.11(0.0$ to 0.22$)$ & 0.22 (0.07 to 0.38 ) & 1.32 (0.94 to 1.69$)$ \\
\hline Prevalence/1000 (95\% CI) & $0.53(0.29$ to 0.77$)$ & $0.20(0.05$ to 0.34$)$ & $0.08(0.0$ to 0.18$)$ & 0.81 (0.52 to 1.11$)$ \\
\hline \multicolumn{5}{|l|}{ Total with PCHI } \\
\hline Number & 90 & 20 & 20 & 130 \\
\hline Prevalence/1000 (95\% Cl) & $2.52(2.0$ to 3.04$)$ & $0.56(0.32$ to 0.81$)$ & $0.56(0.32$ to 0.81$)$ & 3.64 (3.02 to 4.27 ) \\
\hline
\end{tabular}

$\mathrm{BHE}$, better hearing ear; $\mathrm{HL}$, hearing level; $\mathrm{PCHI}$, permanent congenital hearing impairment; WHE, worse hearing ear. 


\section{Factors affecting the prevalence in early childhood}

Of the 130 PCHIs present in primary school, 90 had been born in Waltham Forest with a congenital impairment (2.52/1000) (table 1). Congenital PCHI was the default classification when there was no indication that the impairment was either acquired or late onset. An additional 20 had moved into the district in early childhood. Twelve had emigrated from Angola, Kosovo, Montserrat, Somalia, the West Indies, Pakistan and countries in Eastern and Western Europe with eight coming from elsewhere in the UK. Another 20 (0.56/1000) had acquired late onset deafness by school age. Nine had moderate or worse late onset bilateral PCHI (0.25/1000), and of them four were deafened as a result of meningitis. The others had syndromic deafness (Pendred and Alstrom) or a known family history. All but one of those with mild late onset bilateral PCHI had persistent impairment related to craniofacial dysmorphology or a syndrome. Of those with UHL only three had acquired their impairment-each as a legacy of meningitis or a childhood infection.

\section{Routes to identification}

Nine of the 20 children moving in had a known deafness that had been identified before coming to the district and because the identification histories were largely unverifiable they were excluded from this analysis. The identification of the remaining 121 is detailed in table 2. The UNHS had remained the most effective route. Although nine children identified by the UNHS had subsequently moved out (almost exactly matching the PCHIs moving in from elsewhere in the UK), 1.79/1000 of those in school had a PCHI identified when they were newborn. The most productive postneonatal case finding was reactive referral to the Paediatric Audiology Service. This route identified 44 of the 57 children not referred neonatally (77\%) and a yield of $1.23 / 1000$. Although the majority had a mild impairment or UHL around one-third (14 of 44) had a moderate or worse bilateral deafness.

\section{Identification by the neonatal screen}

UNHS had failed to identify four of the 36 with moderate or worse congenital deafness. One had not completed the screen. Another had a region of near normal hearing over the high frequencies and had retained her cochlear emissions. Another two had ANSD-one from severe perinatal hypoxia but the other with genetic ANSD. The UNHS programme sensitivity for this severity of deafness was therefore $89 \%$.

Unsurprisingly, programme sensitivity was lower for mild impairments. Of the 35 cases, where there was no indication that the mild impairment was either acquired or late onset, only 17 had their PCHI confirmed by UNHS (sensitivity of $49 \%$ ). However, of the remaining 18, 3 had not completed the programme but only 5 had passed the TEAOE. Ten had failed the TEOAE but there remained diagnostic uncertainty following the ABR because most of the ears (56\%) failing the TEOAE had an $\mathrm{ABR}$ threshold $\leq 40 \mathrm{~dB} \mathrm{nHL}(0 \mathrm{~dB} \mathrm{nHL}$ attenuator setting was 32.8 peak to peak equivalent sound pressure level) (figure 1).

The same difficulties were experienced in those with UHL. Of the 19 cases with congenital UHL, 15 were identified by the UNHS (programme sensitivity of $79 \%$ ) and of the 4 missed 3 had mild unilateral impairment where once again there had been diagnostic uncertainties. The remaining child had not been enrolled for the UNHS.

\section{The ages of referral and confirmation}

The referral and confirmation ages are detailed in table 3 . The nine children moving in with unverified histories were excluded. Of the 64 school children where PCHI had been picked up at birth, the 75th centile age of confirmation was just below 4 months. However, for those identified by postneonatal pathways the 75 th centile age of both referral and confirmation was $>5$ years.

\section{Comparison with national data}

Although dwarfed by the UK ascertainment and Trent studies, the prevalence of moderate or worse bilateral deafness identified in Waltham Forest was not significantly different to that ascertained in the other two studies ( $p>0.1)$. The larger cohorts offered no prevalence data on mild or unilateral deafness (table 4).

\section{DISCUSSION}

Now that the NHSP has been successfully rolled out in the UK, the need for postneonatal care pathways has become evident. This is particularly pertinent for the paediatricians who will lead the local Children's Hearing Services and

Table 2 Case identification of the PCHls present in primary school $(n=121)$

\begin{tabular}{|c|c|c|c|c|}
\hline & \multicolumn{4}{|l|}{ PCHIs identified } \\
\hline & Moderate and worse bilateral (BHE) & Mild bilateral (BHE) & Unilateral (WHE) & All degrees \\
\hline \multicolumn{5}{|l|}{ Neonatal screen } \\
\hline Number & 32 & 17 & 15 & 64 \\
\hline Yield/1000 (95\% Cl) & $0.9(0.59$ to 1.21$)$ & $0.48(0.25$ to 0.7$)$ & $0.42(0.21$ to 0.63$)$ & $1.79(1.36$ to 2.23$)$ \\
\hline \multicolumn{5}{|l|}{ Concerns $0-1$ year } \\
\hline Number & 3 & 1 & 0 & 4 \\
\hline Yield/1000 (95\% Cl) & 0.08 (0 to 0.18$)$ & 0.03 (0 to 0.08$)$ & $0(0)$ & $0.11(0.0$ to 0.22$)$ \\
\hline \multicolumn{5}{|c|}{ Health visitor distraction test } \\
\hline Number & 1 & 1 & 0 & 2 \\
\hline Yield/1000 (95\% Cl) & $0.03(0$ to 0.08$)$ & $0.03(0$ to 0.08$)$ & $0(0)$ & $0.06(0.0$ to 0.13$)$ \\
\hline \multicolumn{5}{|l|}{ Concerns $1-5$ years } \\
\hline Number & 11 & 22 & 7 & 40 \\
\hline Yield/1000 (95\% Cl) & $0.31(0.13$ to 0.49$)$ & $0.62(0.36$ to 0.87$)$ & $0.2(0.05$ to 0.34$)$ & $1.12(0.77$ to 1.47$)$ \\
\hline \multicolumn{5}{|l|}{ School entry sweep test } \\
\hline Number & 3 & 4 & 4 & 11 \\
\hline Yield/1000 (95\% CI) & $0.08(0$ to 0.18$)$ & 0.11 (0 to 0.22 ) & 0.11 (0 to 0.22 ) & $0.31(0.13$ to 0.49$)$ \\
\hline
\end{tabular}

$\mathrm{BHE}$, better hearing ear; $\mathrm{PCHI}$, permanent congenital hearing impairment; WHE, worse hearing ear. 
have responsibility for PCHI not identified by the newborn screen. ${ }^{6}$

There have been alerts that this may not be an inconsiderable undertaking. The Wessex controlled trial of UNHS warned that even with high test sensitivity, only $56 \%$ of those in school with moderate or worse bilateral PCHI had been identified as newborns. ${ }^{1}$ There is a good evidence base supporting the earliest management of children with this degree of deafness and their identification is the 'major' aim of NHSP. However, entirely similar results have now been achieved from the larger 10-year Waltham Forest cohort with only $59 \%$ of school children having been identified by a neonatal screen which was $89 \%$ sensitive to this degree of PCHI. In part, this lack of sensitivity could be attributed to the inability of the two-stage TEOAE screen to identify ANSD, but the prevalence of this condition at 1/10000 is ${ }_{10}{ }^{8}$ and as reported in the current cohort not all cases are identifiable neonatally. The implication is that even when a neonatal screen is sensitively implemented around 1 in 10 of those with moderate or worse congenital PCHI will require detection by postneonatal care pathways. Such pathways are also required for around $0.25 / 1000$ children with late onset or acquired deafness.

Deaf children also move into a district and when the Whipps Cross UNHS was undertaken they had rarely received a neonatal hearing test. Today, almost all moving around the UK

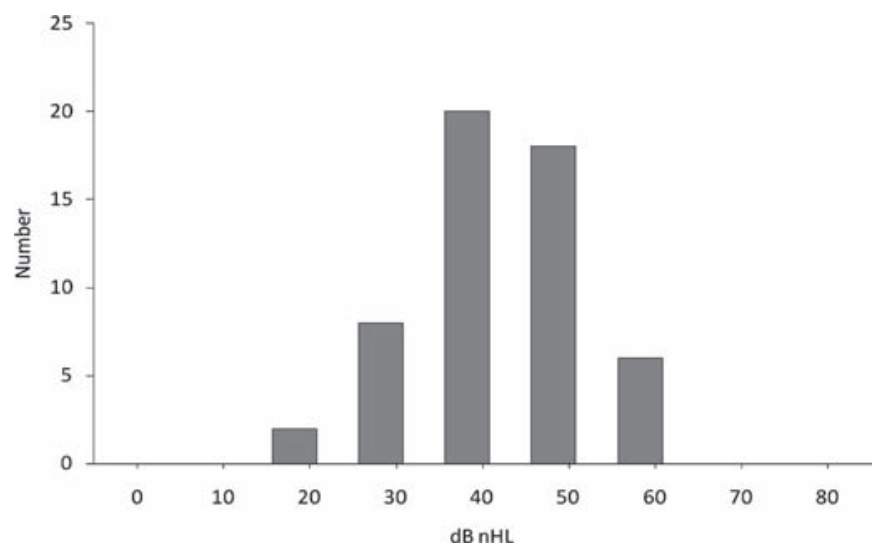

Figure 1 The auditory brainstem response (ABR) thresholds by ear for those with mild permanent congenital hearing impairment who had neonatal $A B R(n=54)$. would have benefited from NHSP enrolment. However, in the reported district in East London the majority moved in from countries where screening was not offered and this continues to pose a problem recognised in the initial cautions about screen expectations. ${ }^{2}$

Late case finding is also necessary for those with milder and unilateral PCHIs. The case for their early detection is not robust but this does not imply that they are without disability and studies undertaken from the 1980s have demonstrated that more than a third will experience academic difficulties. ${ }^{14}$ In the current cohort over $40 \%$ had an ABR threshold of 50 or $60 \mathrm{~dB} \mathrm{nHL}$, and over half had been fitted with hearing aids by school age. However, because not all experience disability, population screening is difficult to justify and rational identification requires reactive pathways. A multicentre evaluation of a two-stage TEOAE/A-ABR confirmed that mild PCHI is missed, ${ }^{15}$ which was consistent with the insensitivity of the neonatal ABR for identifying this degree of PCHI in the reported cohort. Postneonatal reactive identification is required. Sensitivity was much higher for congenital UHL with only 2 in every 10 missed neonatally. Previously, many cases of UHL required identification later in childhood because they were acquired from childhood infections. ${ }^{16}$ However, in the current cohort less than $1 / 10000$ had acquired UHL and in none was it related to mumps.

Follow-up of the 10-year cohort supported the worth of screening the hearing at birth. Three-quarters of those so identified had their deafness confirmed below 4 months of age. However, those referred postneonatally fared less well and more than one-quarter were already in school before deafness was confirmed. These delays re-enforce the challenges to services. Those children not identified at birth often present with subtle difficulties with hearing impairments that are far from obvious. The success of the newborn programme has rightly been acclaimed but it cannot 'stand alone' and postneonatal care pathways remain an essential children's hearing service.

Funding There has been no funding related to this article or to the longitudinal follow-up of the Waltham Forest cohort.

Competing interests None.

Provenance and peer review Not commissioned; externally peer reviewed.

Table 3 The referral and confirmation ages for those with PCHI in primary school $(n=121)$

\begin{tabular}{|c|c|c|c|c|c|c|}
\hline \multirow[b]{2}{*}{ Degree of PCHI } & \multicolumn{3}{|c|}{$\begin{array}{l}\text { Median ages in weeks (IOR) when } \\
\text { identified by UNHS }\end{array}$} & \multicolumn{3}{|c|}{$\begin{array}{l}\text { Median ages in weeks (IOR) when } \\
\text { identified postneonatally }\end{array}$} \\
\hline & $\mathrm{n}$ & Referral & Confirmed & $n$ & Referral & Confirmed \\
\hline Mild bilateral & 17 & $5(4-7)$ & $12(10-13)$ & 28 & 195 (131-239) & $215(161-259)$ \\
\hline Moderate and worse bilateral & 32 & $6(5-9)$ & $10(8-16)$ & 18 & $166(110-241)$ & $188(112-255)$ \\
\hline UHL & 15 & $6(2-8)$ & $11(10-15)$ & 11 & 248 (239-272) & $254(244-281)$ \\
\hline All & 64 & $6(4-8)$ & $11(9-15)$ & 57 & $201(142-269)$ & 223 (167-274) \\
\hline
\end{tabular}

$\mathrm{PCHI}$, permanent congenital hearing impairment; UHL, unilateral hearing loss; UNHS, universal neonatal hearing screens.

Table 4 Comparisons of Waltham Forest prevalence of moderate and worse PCHI with national data

\begin{tabular}{|c|c|c|c|c|c|}
\hline & Report & Cohort period & Population & Prevalence or yield/1000 (95\% CI) & Pearson's $\chi^{2}$ \\
\hline \multirow[t]{3}{*}{ Prevalence from cohort studies } & Waltham Forest & $1992-2002$ & 35668 & Bilateral $1.51 / 1000$ (1.11 to 1.92 ) & NA \\
\hline & MRC Trent study & $1985-1990$ & 366480 & Bilateral $1.33 / 1000$ (1.22 to 1.46$)$ & $0.829 *$ \\
\hline & UK national PCHI ascertainment & $1988-1993$ & 4697700 & Bilateral $1.44 / 1000$ (1.41 to 1.48$)$ & $0.128^{*}$ \\
\hline
\end{tabular}

${ }^{*} \mathrm{p}>0.10$.

MRC, Medical Research Council; PCHI, permanent congenital hearing impairment. 


\section{REFERENCES}

1. Kennedy C, McCann D. Universal neonatal hearing screening moving from evidence to practice. Arch Dis Child Fetal Neonatal Ed 2004;89:F378-83.

2. Russ S. Measuring the prevalence of permanent childhood hearing impairment. BMJ 2001;323:525-6.

3. Watkin PM, Baldwin M. Confirmation of deafness in infancy. Arch Dis Child 1999:81:380-9.

4. Kennedy CR. Controlled trial of universal neonatal screening for early identification of permanent childhood hearing impairment: coverage, positive predictive value, effect on mothers and incremental yield. Wessex Universal Neonatal Screening Trial Group. Acta Paediatr Supp/ 1999;88:73-5.

5. Fortnum HM, Summerfield AQ, Marshall DH, et al. Prevalence of permanent childhood hearing impairment in the United Kingdom and implications for universal neonatal hearing screening: questionnaire based ascertainment study. BMJ 2001;323:536-40.

6. Bamford J, Fortnum H, Bristow K, et al. Current practice, accuracy, effectiveness and cost-effectiveness of the school entry hearing screen. Health Technol Assess 2007;11:1-168, iii-iv.

7. Department of Health. Transforming services for children with hearing difficulty and their families: a good practice guide. DH Publications 2008:1-44.

8. Hall D. Children's audiology services - a multi-professional review in partnership with the NHSU. http://www.rcpch.ac.uk/doc.aspx?id Resource=1524 (Accessed 31 January 2010).
9. Watkin PM. Outcomes of neonatal screening for hearing loss by otoacoustic emission. Arch Dis Child Fetal Neonatal Ed 1996;75:F158-68.

10. Davis A, Bamford J, Wilson I, et al. A critical review of the role of neonatal hearing screening in the detection of congenital hearing impairment. Health Technol Assess 1997:1:i-iv, 1-176.

11. Kennedy C, Kimm L, Thornton R, et al. False positives in universal neonatal screening for permanent childhood hearing impairment. Lancet 2000;356:1903-4.

12. Starr A, Picton TW, Sininger Y, et al. Auditory neuropathy. Brain 1996;119 (Pt 3):741-53.

13. Fortnum HM, Davis A, Butler A, Stevens J. Health Service Implications of Changes in Aetiology and Referral Patterns of Hearing Impaired Children in Trent 1985-1993. Report to Trent Health. Nottingham/ Sheffield: MRC institute of Hearing Research and Trent Health.

14. Tharpe AM. Unilateral and mild bilateral hearing loss in children: past and current perspectives. Trends Amplif 2008;12:7-15.

15. Johnson JL, White KR, Widen JE, et al. A multicenter evaluation of how many infants with permanent hearing loss pass a two-stage otoacoustic emissions/ automated auditory brainstem response newborn hearing screening protocol. Pediatrics 2005;116:663-72.

16. Bess FH, Klee T, Culbertson JL. Identification, assessment, and management of children with unilateral sensorineural hearing loss. Ear Hear 1986; 7:43-51.

\section{Glossary}

Congenital impairment

Acquired impairment Late onset impairment Progressive impairment Confirmation of impairment Confirmation with audiological certainty

Referral
Hearing impairment considered to be present and detectable at or very early after birth, including those babies with an early or perinatally acquired impairment. This was the default classification when there was no indication that the impairment was either acquired or late onset

Hearing impairment that first manifested itself postnatally and could be attributed to an identifiable exogenous cause Hearing impairment that first manifested itself postnatally but could not be attributed to an identifiable exogenous cause Hearing impairment that may be congenital, acquired or late onset but which worsened over time The process of establishing the presence of hearing impairment by an age-appropriate threshold test with high reliability of the results Confirming the presence of hearing impairment by age-appropriate tests where both the degree and type of impairment were established

The process of referring a child to an audiologist for an assessment of hearing threshold either reactively because of professional or parental concern or from a hearing screen. When used as an indicator of outcome only referrals resulting in confirmation were included 